\title{
Surgically Assisted Rapid Palatal Expansion (SARPE): A Literature Review
}

\author{
Sharath Kumar Shetty ${ }^{1}$, Neeraj N. S ${ }^{2 *}$, Mahesh Kumar $\mathrm{Y}^{3}$, Vijayananda K. Madhur ${ }^{4}$
}

${ }^{1}$ Professor \& HOD, Department of Orthodontics \& Dentofacial Orthopaedics, K.V.G Dental College \& Hospital, Sullia, Karnataka, India

${ }^{2}$ Post Graduate Student, Department of Orthodontics \& Dentofacial Orthopaedics, K.V.G Dental College \& Hospital, Sullia, Karnataka, India

${ }^{3}$ Professor, Department of Orthodontics \& Dentofacial Orthopaedics, K.V.G Dental College \& Hospital, Sullia, Karnataka, India

${ }^{4}$ Reader, Department of Orthodontics \& Dentofacial Orthopaedics, K.V.G Dental College \& Hospital, Sullia, Karnataka, India

DOI: $10.36347 /$ sjds.2021.v08i01.005

| Received: 18.12.2020 | Accepted: 05.01.2021 | Published: 09.01.2021

*Corresponding author: Neeraj N. S

Abstract

Review Article

Transverse maxillary hypoplasia, in adolescents and adults, is frequently seen in nonsyndromal and syndromal patients including cleft patients. In skeletally matured patients, the uni or bilateral transverse hypoplasia can be corrected by means of a surgically assisted rapid maxillary expansion. The treatment is a combination of orthodontics and surgical procedures and provides dental arch space for alignment of teeth. The procedure also causes a substantial enlargement of the maxillary apical base and of the palatal vault, providing space for the tongue for correct swallowing and thus preventing relapse. In addition, a distinct subjective improvement in nasal breathing associated with enlargement of the nasal valve towards normal values is seen with an increase of nasal volume in all compartments. A review of SARPE in general has been highlighted in this article.

Keywords: hypoplasia, adults, nasal breathing, SARPE.

Copyright $(\mathcal{O} 2021$ The Author(s): This is an open-access article distributed under the terms of the Creative Commons Attribution 4.0 International License (CC BY-NC 4.0) which permits unrestricted use, distribution, and reproduction in any medium for non-commercial use provided the original author and source are credited.

\section{INTRODUCTION}

A correct transverse skeletal relationship between the jaws is essential for stable and functional occlusion [1]. Maxillary transverse deficiency (MTD) is one of the most pervasive and common skeletal problems in the craniofacial region, often combined with a simultaneous vertical or antero-posterior skeletal discrepancy [2]. MTD is prevalent in both syndromic and non-syndromic patients [3].

The most frequently reported clinical manifestations are unilateral or bilateral posterior crossbites, palatal inclination of teeth, dental crowding, high palatal arch, narrow, tapering arch form and problems associated with nasal breathing [4]. Unlike vertical or sagittal discrepancies, MTD is difficult to diagnose extraorally. The extraoral manifestations are often discrete, uncertain and limited to narrow alar bases, paranasal hollowing and a deep nasolabial groove. Vertical and sagittal anomalies often exist concomitantly; as they are more recognizable they will clinically mask the extraoral appearance of a MTD.

The etiology of MTD is multifactorial, including congenital, genetic, developmental, traumatic or iatrogenic factors. Examples of causative factors are different syndromes, thumb and finger-sucking habits, mouth breathing during critical growth periods, trauma or iatrogenic injuries after cleft palate repair [5-7].

The prevalence of MTD is reported to be 8.5 to 22 per cent. The wide range of prevalence can be attributed to lack of uniformity in classification of maxillary transverse deficiency, such as magnitude of the skeletal discrepancy and the severity of dental components. There is no difference in prevalence with respect to gender or ethnicity and no available data in the literature on prevalence in an adult skeletally mature population $[8,9]$.

It is essential to distinguish between skeletal and dental components of the deformity in order to select the treatment modality which will achieve a stable, functional result [10]. The maxillary constriction can be purely skeletal, purely dental or a combination of both. Some cases have an apparent maxillary deficiency due to the palatal inclination of one or two posterior teeth. These maxillary transverse deficiencies with purely dental components are, in most cases, simple orthodontic problems and do not require extensive orthodontic or surgical treatment [11]. 
Jacobs et al., stated that skeletal MTD can be divided into two categories; real and relative. Relative MTD implies that a transverse discrepancy exists clinically, but is attributable to a sagittal discrepancy between the jaws, i.e. in a relative MTD no transverse deficiency exists when the study models are examined in a Class I relationship. This is a common phenomenon in Angle Class III skeletal malocclusions [12].

Bishara and Staley advocated a clinical examination of MTD. The examination takes into account the magnitude of the transverse discrepancy between maxilla and mandible, the number of teeth involved and the initial angulation of the maxillary molars and premolars. A transverse discrepancy exceeding $4 \mathrm{~mm}$ and or buccally inclined maxillary molars and premolars indicate a true skeletal MTD [10].

Real MTD implies a true transverse maxillary insufficiency. Clinically there may or may not be a posterior crossbite. In contrast to relative MTD, true MTD shows a uni or bilateral posterior crossbite when the study models are positioned in a Class I relationship. Real MTD is frequently associated with skeletal Class II malocclusions and skeletal open bites. Although relative MDT can be treated with midpalatal suture opening, dental maxillary transverse deficiency and relative MTD require no orthopaedic or surgical transverse expansion. In such cases, the transverse discrepancy can be corrected by conventional orthodontics, with or without extractions. In surgical treatment of skeletal sagittal anomalies, relative MTD will be corrected by the following sagittal displacement. Real MTD, however, requires opening of the midpalatal suture and separation of the maxilla to normalize the transverse deficiency and cannot be achieved by conventional orthodontics alone. Once the diagnosis has been made and a need for expansion is ascertained, other factors must be addressed, such as the magnitude of the transverse discrepancy, the age of patient, whether the expansion should be achieved orthopaedically and or by surgical intervention [13].

\section{History of Orthodontic Treatment for Maxillary Constriction}

Growth at the suture occurs through deposition of new bone at the sutural margin by the adjacent cellular layer. Toward the end of fetal life the cellular layers decrease in thickness, indicating that the rate of growth is slowing down, and the number of fibers in the intermediate layer uniting the capsular layers decreases. In a study of human sutures from birth to 18 years, Latham and Burston concluded that after about 2 of 3 years the sutures of the skull in general functioned primarily as sites of union of bones, but localized remodelling is a continuing process. Cranial sutures are unified before complete eruption of the third molar. Soon after this, facial sutures close, and the sutures connecting the cranial and facial complexes are the last to close $[14,15]$. Regarding the facial sutures, Sicher
[16] states that the closure of sutures in human beings starts, as a rule, in the middle 30 s at the posterior end of the median palatine suture but that some facial sutures, including the frontozygomatic, may remain open even in older age groups. This view is supported by Wright, who claimed the intermaxillary and palatine sutures to be unossified and susceptible to comparatively easy separation at as late an age as 35 years. A conflicting view is expressed by Persson [17], who found evidence of bony union at 17 years in the midpalatal suture. Latham and Burston [14], however, found no evidence of synostosis in the same suture by the age of 18 years. An over-all view is expressed by Scott [18], who believes that, although most facial sutures appear open on the surface of old skulls, some degree of union may be present in the substance of the suture. It is obvious therefore, that the available literature is inconclusive and conflicting. In clinical practice, skeletal correction of the transverse discrepancy via orthodontics (orthopaedics) is successful until the age of approximately 14-15 years depending on the gender of the patient. After this age, orthodontic widening becomes virtually impossible and very painful $[19,20]$. In general, it is assumed that closure of the midpalatal suture prevents this type of expansion [21]. In the first part of nineteenth century, Lefoulon [22] and Talma [23] reported on maxillary expansion with a palatal or buccal C-shaped spring. A method, reserved for less severe cases, consisted of lateral thumb pressure, 'every morning and even many times daily', by the parent or the child itself. The first documented case of orthodontic correction of maxillary width discrepancies was by Angell [24]. He performed rapid maxillary expansion with the use of a jackscrew appliance in a 14year-old girl. He observed that by turning the jackscrew daily, he was able to open the maxillary suture sufficiently in a period of 2 weeks. Angell mentions correction of maxillary width discrepancies by opening the midpalatal suture. In 1913, Schröder-Benseler [25] presented the still-popular all-wire frame with a nonspring-loaded jackscrew, the hygienic appliance. Derichsweiler uses bonds to the premolar and molar, which are embedded into a split acrylic base plate with an incorporated conventional orthodontic expansion screw. In 1961 Haas 'Reintroduced' rapid maxillary expansion (RME) and mentions in 1970 that the use of RME is ideally during the growth spurt. Reichenbach \& Brück published an excellent survey on orthodontic treatment of maxillary transverse hypoplasia in 1967 [26].

\section{Surgically Assisted Rapid Maxillary Expansion}

Surgically assisted rapid maxillary expansion SARME is a form of distraction osteogenesis (DO). In the purest sense, craniofacial DO was first reported in the early 1860's by Angell long before the biological healing principles of DO were known. DO involves the process of generating new bone in a gap between two bone segments, in which new bone is a result of tensile stress across the bone gap [27, 28]. The technique was 
first described in 1905 by Codivilla [29] but remained undeveloped until Ilizarov "rediscovered" the technique in the 1950's. The unique feature of DO is stability and the biological concept of simultaneous expansion of a soft tissue matrix, including blood vessels, nerves, muscles, mucosa and periosteum $[30,31]$. The principle of DO is based on four phases; osteotomy or surgical phase, a latency period, a distraction period and finally a consolidation period. The initial surgery and osteotomy is followed by a latency period of between five and seven days. This is a period of rest and formation of a fibro vascular haematoma; newly formed capillaries and granulation tissue infiltrate into the fibrin clot. Shorter latency periods are generally associated with decreased callus formation and inadequate osteogenesis, whereas longer latency periods are usually associated with premature consolidations [32].

In the following distraction phase, collagen fibres are formed parallel to the distraction vector; intramembranous ossification starts and follows the collagens fibres towards the midline. Further mineralisation and remodelling of the immature soft bone takes place during the consolidation phase. Bone remodelling begins during the consolidation phase and continues over 1-2 years, eventually transforming the regenerated tissue into a mature osseous structure, similar in size and shape to the adjacent bone [33, 34]. SARME is far from a standardized procedure. When first described by Brown, as a method to correct MTD in non- growing individuals, only midpalatal splitting was involved. The rationale for choosing a particular osteotomy technique is, as mentioned above, based on the assumption of different skeletal resistance in the maxilla. Those who consider the inter maxillary suture to be the essential area of resistance recommend paramedial palatal osteotomies [35, 36], whereas those who regard the zygomatico maxillary buttress as the main area of resistance advocate osteotomy solely in the lateral areas of the maxilla [37]. Some include the pterygomaxillary complex in the lateral osteotomies [38]. Many clinicians advocate combined osteotomies in the palatal, anterior and lateral maxilla and especially posteriorly at the pterygomaxillary complex. Thus, there is no gold standard for optimal surgical procedures and no general consensus in the literature with respect to skeletal effects after SARME [39].

\section{History of surgical treatment for maxillary constriction}

Once skeletal maturity has been reached, orthodontic treatment alone cannot provide a stable widening of the constricted maxilla in cases of deficiencies of more than $5 \mathrm{~mm}$. In general, an orthodontist can camouflage transverse discrepancies less than $5 \mathrm{~mm}$ with orthopaedic forces alone [41].

Tooth extractions for alignment of dental arches are often unnecessary. Brown [42] probably first described a technique of SARME with midpalatal splitting in his textbook. Heiss [43] probably first inaugurated the midline splitting in the anterior maxilla for the extension of the compressed maxillary arch for orthodontic reasons. In 1961, Haas described the downward and forward movement of the maxilla that occurs during RME because of the location of the Cranio Maxillofacial sutures. He believed that the maxillary halves separated from each other rather in a tipping than in a parallel fashion due to the strength of the zygomatic buttresses. Isaacson \& Ingram [44] mention that historically, the midpalatal suture was thought to be the area of resistance to expansion, but the facial skeleton increases its resistance toexpansion as it ages and matures, and that the major site of resistance is not the midpalatal suture but the remaining maxillary articulations. Wertz [45] advocated that resistance of the zygomatic arch prevents parallel opening of the midpalatal suture. In 1975, Lines and in 1976 Bell \& Epker demonstrated that the area of increased facial skeletal resistance to expansion was indeed not the midpalatal suture, but the zygomaticotemporal, zygomaticofrontal and zygomaticomaxillary sutures. Identification of these areas of resistance in the craniofacial skeleton stimulated the development of various maxillary osteotomies to expand the maxilla laterally in conjunction with orthodontic RME appliances4. The areas of resistance to lateral forces in the midface are the piriform aperture (anterior), the zygomatic buttress (lateral), the pterygoid junction (posterior) and the midpalatal synostosed suture (median). In the early reports all four are transsected. In 1972 Steinhauser [46] reports a maxillary expansion osteotomy technique without the use of distraction, a Le Fort I type of osteotomy in combination with the surgical splitting of the palate in the midline, after which a triangular unicortical iliac graft is inserted into the void created by the expansion. More recently, with the emphasis on decreased morbidity and ambulatory surgery, fewer supports are osteotomized; the anterior, lateral and median, the lateral and median, the anterior, posterior and lateral, the anterior and lateral. Most reports note that surgically assisted maxillary expansion is more stable than orthodontic RME alone. Glassmann et al., Alpern \& Yurosko and Lehmann \& Haas reported successful expansion in humans performed with a Hyrax appliance following a lateral osteotomy from the piriform rim to the pterygoid plate without palatal surgery [47, 48]. Their study did not consider the amount of skeletal versus dental expansion and the corresponding relapse following a retention period. In 1984 Glassmann et al. postulates that uniform palatal expansion can be achieved without sectioning of either palate or the pterygomaxillary fissure [49].

In the year 1999, Mommaerts [50] presented the Trans Palatal Distractor (TPD), which is a boneborne device for SARME. After surgical release of the areas of maxillary support the tooth-borne devices used for SARME cause undesired movements of the 
Sharath Kumar Shetty et al., Sch J Dent Sci, Jan, 2021; 8(1): 25-40

abutment teeth during expansion and retention phases that could lead to periodontal problems. Prolonged retention and overcorrection is advisable to counteract skeletal relapse. The TPD avoids all of these aforementioned problems, since fixation is sought in palatal bone. Recently, the Magdenburg Palatal Distractor (PD) was presented, also a bone-borne device which claims to have no relapse [51].

\section{History of Distraction}

As mentioned before SARME is a form of distraction that was applied before its biological healing principles were known. Codivilla was the first to describe the technique of distraction osteogenesis for the shortened femur in 1905. Ilizarov described the use of distraction osteogenesis in the field of Orthopaedics to lengthen the leg bones in a large group of patients in 1990. The technique is based on a 5-day period of rest after corticotomy before the expansion starts. This gives the tissue time to form the first callus but is too short for consolidation. Four phases of new bone formation can be described. The first is a fibro vascular haematoma; between day 5 and 7 collagen fibers are formed that will arrange parallel to the distraction vector. Second, the bone formation follows the collagen fibers through intramembranous ossification; from the outside to the inside. Third, remodeling phase of the new bone. Fourth, formation of solid compact bone with the same texture as the surrounding (old) bones. When the distraction is performed too fast, the collagen fibers might lose contact and there is no in growth of new bone, providing non- or mal-union. In cases of a too slow distraction premature consolidation can occur and the requested elongation cannot be reached $[52,53]$.

\section{Areas of Skeletal Resistance}

Various surgical procedures have been developed for SARME in proportion to the primary areas of resistance in the craniofacial skeleton. It was early assumed that the mid-palatal suture was the main area of resistance. Surgical techniques favouring midpalatal osteotomies are derived from Timms [36] histological studies in the sixties. Isaccsson and Kennedy et al., concluded that the major resistance to maxillary expansion was not the midpalatal suture but the remainder of the maxillary articulations [44]. Wertz [45] stated that resistance of the zygomatic arch prevented parallel opening of the midpalatal suture, which was highlighted by Lines and Bell and Epker's results [54, 55]. On the basis of photoelastic observations, Shetty et al., insisted that the mid-palatal suture and the pterygomaxillary region were the most resistant areas and exclusive use of bilateral zygomatic buttress osteotomies was inadequate. In threedimensional FEM studies Jafari et al,, showed high resistance posteriorly, and particularity at the sphenoid and zygomatic bones and concluded a need for surgical release in this area. Holberg and Rudzki Janson reported lateral bending of the pterygoid process and increased stress in the sphenoidal area in adulthood, after maxillary expansion [56].

\section{Indications for SARPE}

There is a lack of consensus among orthodontists and surgeons about the indications for SARPE. Although maxillary expansion might be required for many patients, an accurate diagnosis of MTD is somewhat ambiguous. The following have been reported in the previous literatures as indications for SARPE, all applying to a skeletally mature patient with a constricted maxillary arch.

1. To increase maxillary arch perimeter, to correct posterior crossbite, and when no additional surgical jaw movements are planned.

2. To widen the maxillary arch as a preliminary procedure, even if further orthognathic surgery is planned. This is to avoid increased risks, inaccuracy, and instability associated with segmental maxillary osteotomy.

3. To provide space for a crowded maxillary dentition when extractions are not indicated.

4. To widen maxillary hypoplasia associated with clefts of the palate.

5. To reduce wide black buccal corridors when smiling.

6. To overcome the resistance of the sutures when OME has failed [57].

\section{Patient Selection}

A thorough review of the literature shows significant disparities among clinicians regarding the criteria for case selection and the indications for SARPE. In this section, we will address the diagnostic procedures that are critical to proper case selection.

\section{Diagnosis}

The first step in the case selection process is determination of MTD. Unlike discrepancies in the vertical and the antero posterior dimensions, diagnosis of MTD is difficult. There is much literature on the various methods used to diagnose this condition. Clinical evaluation, model analysis, occlusograms, and radiographic measurements have been recommended for an accurate assessment. Clinical evaluation includes assessment of the maxillary arch form and symmetry, shape of the palatal vault, width of the buccal corridors on smiling, occlusion, and predominant mode of breathing (nasal or oral). Excessively wide buccal corridors, para nasal hollowing, or narrow alar bases usually suggest MTD. The soft-tissue thickness should also be evaluated because it can mask MTD. Unilateral or bilateral crossbite, severe crowding, a V-shaped or an hourglass shaped occlusion, and a high palatal vault are additional visual parameters that can help the clinician make the first determination of MTD in a patient. Another factor that needs assessment is a mandibular shift on closure. This can often be a chin deviation with a unilateral crossbite. To identify the nature of a shift, it might be necessary to use a muscle deprogramming 
device suchas a bite plate for a few days. These devices are needed more often for adults whose muscular kinesthetic memory and proprioceptive influences are ingrained. Such a deprogramming device allows the muscles to move the mandible in coordinated function that is undisturbed by deflective tooth contacts [58, 59]. Another aspect that needs determination is whether the MTD is relative or absolute. This is essential in the evaluation of sagittal discrepancies (especially Class III malocclusion). An attempt is made to articulate and align the models in Angle Class I molar and canine relationship to evaluate arch coordination. Relative MTD implies that the apparent deficiency is the result of the discrepancy of the maxilla or both jaws in the sagittal plane. Absolute MTD implies a true horizontal width insufficiency [60]. Study models should be used to thoroughly assess the arch form and the shape and make specific measurements to evaluate for MTD. Several indexes have been proposed by various authors to measure lateral discrepancies. The most common include the indexes of Pont, Linder-Harth, and Korkhaus [61]. Although these indexes offer a guide to diagnose MTD, they are population specific and not completely reliable. With the advent of digital models in routine clinical practice, additional tools can be used to evaluate arch form and tooth inclinations [62]. The evaluation of the buccolingual inclination of the posterior teeth is an essential part of the diagnosis. This allows a more accurate distinction between dental and apical base skeletal MTD. The digital models can be viewed in desired cross-sections that permit better visualization of the buccolingual inclination of the teeth. The digital models can also generate images for occlusograms whereby the coordination of the maxillary and mandibular arches can be evaluated. They provide occlusal simulations and assist in the diagnosis of relative or absolute MTD. Lehman et al., [63] recommended a palatal or an occlusal radiograph as an essential tool to evaluate the ossification of the midpalatal suture. This, however, is unreliable because of the superimposition of other bony structures on the midpalatal suture and the lack of adequate visualization of the posterior part of the intermaxillary suture. This is relevant because histologic studies have shown that obliteration of the suture is more common in the posterior region of the intermaxillary suture. The value of an occlusal radiograph is also unclear, since studies have shown that the midpalatal suture does not offer much resistance to expansion [64].

Betts et al., [2] suggested that posteroanterior cephalograms are the most readily available and reliable means to identify and evaluate transverse skeletal discrepancies between the maxilla and the mandible. Using cephalometric landmarks as described by Ricketts [65], they presented 2 methods for quantification of the MTD: maxillomandibular width differential and maxillomandibular transverse differential index. These methods have been criticized because the transverse discrepancy between the maxilla and the mandible is measured on bony landmarks that are greatly separated from the dentition and the apical bases. The advent of 3-dimensional imaging techniques is the most recent tool for diagnosis that have enabled an accurate visualization of the craniofacial region. It allows for evaluation of the spatial relationships of various areas of the jaws. Cone-beam computed tomography can generate scans that enable the clinician to perform a 3-dimensional evaluation of the apical bases including horizontal sections of the apical bases at different levels. These images can help the clinician to make an accurate and detailed analysis of the nature and location of the discrepancy including asymmetries [66].

\section{Age as Criterion}

The patient's age has been considered by most authors and clinicians as the fundamental basis for distinguishing the use of OME vs SARPE to treat MTD. However, conflicting views regarding when OME is successful and when to request surgical assistance for treating MTD are found in the literature. Epker and Wolford [67] recommended surgical assistance for maxillary expansion in patients over 16 years of age. Timms and Vero [36] used 25 years as the upper limit for recommending OME. Mossaz et al., [68] arbitrarily recommended "after the second decade of life" for surgical assistance of maxillary expansion. Mommaerts stated that OME is indicated for patients younger than 12 years, and, for those over 14 years, corticotomies are essential to release the areas of resistance to expansion. Alpern and Yurosko suggested that sex should also be considered as a selection criterion. According to them, men over the age of 25 and women over 20 require surgical assistance for expansion. Further confusion is added by several case reports in which OME has been shown to be successful in much older adults [69]. These authors suggested that, although an orthopedic effect was not observed, slow expansion results in a combination of membranous warpage and some sutural stretching to provide the desired end result. They also suggested that slow expansion might not be as kind to the gingivae, but it is clinically adequate and stable. Determination of skeletal age is an important parameter for case selection. It is possible that chronologically advanced patients in case reports whose OME was successful were skeletally immature. The reverse can also be true in chronologically younger patients with advanced skeletal maturity whose OME might be unsuccessful [70].

\section{Medical History}

In treatment planning and case selection for MTD, the patient's medical condition must be thoroughly evaluated. Investigations on cadaver skulls by Persson and Thilander [71] showed that ossification of the midpalatal suture has wide variations in various age groups. Since OME depends on the sutural patency and the flexibility of the craniofacial skeleton to adapt to controlled mechanical forces, it is essential to evaluate for medical conditions that can influence the 
results of OME. Several metabolic conditions have been linked to sutural synostoses. These include hyperthyroidism, hypophosphatemic vitamin Dresistant rickets, and mucopolysaccharidoses and mucolipidoses.A common link in all these conditions is an underlying abnormality in bone metabolism. The medical history must be carefully evaluated, since developmental dynamics and environmental influences can affect the ability of a suture to respond to external force application. OME would either be unsuccessful or have unfavourable consequences as discussed earlier even in a chronologically young patient with such medical conditions. Synostosis in any of these metabolic disorders can be either simple or complex. Simple synostosis involves fusion of 1 suture, but craniosynostosis syndromes and metabolic disorders are associated with complex synostosis. Individual variability with regard to fusion of sutures is significant. Recent evidence from molecular biology has shed light on the underlying mechanisms of suture fusion. These findings might have significant implications on the selection of treatment. Bony obliteration of the suture site is caused by premature or accelerated bone formation in the fibrous suture matrix [72].

This can occur by increasing cell numbers, leading to increased cell density and inducing bony differentiation, or by directly inducing premature differentiation of cells. Cell numbers can be increased by stimulating cell proliferation or by inhibiting apoptosis. These cellular functions are controlled by various growth and transcription factors acting in concert or in parallel with each other. Several growth and transcription factors have been shown to play a role in regulating suture morphogenesis and patency, and, in many instances, the mechanisms by which they do so have begun to be elucidated. It can be hypothesized that a detailed decision making. A detailed medical evaluation is also necessary from the standpoint of general anesthesia that would otherwise preclude the patient from elective surgery [73].

\section{Amount of Expansion}

Betts et al., and others have recommended that the amount of desired expansion is an important factor in case selection for maxillary expansion in adults. In general, an orthodontist can camouflage transverse maxillomandibular discrepancies less than $5 \mathrm{~mm}$ with orthopedic or orthodontic forces alone. When the MTD is greater than $5 \mathrm{~mm}$, surgical assistance is essential. Although both SARPE and segmental osteotomy are used for surgically assisted maxillary expansion, segmental osteotomy is reported to be unstable, especially when more than $8 \mathrm{~mm}$ expansion is desired. It is also essential to evaluate the buccolingual inclination of the teeth because that may either mask or aggravate the discrepancy at the apical bases [2].

\section{Two-Stage vs Singular Surgery}

Surgical correction of MTD may be achieved by either segmental osteotomy or SARPE. Segmental osteotomy is the preferred choice for correction of MTD when a single surgical procedure is planned to correct all maxillo-mandibular discrepancies. Vertical and sagittal repositioning of the maxilla and the mandible can be done at the same time when correction of MTD is done with segmental osteotomy. On the other hand, correction of MTD is done as a first step with that SARPE should be used for patients with an isolated transverse deficiency when OME is not indicated, or with unilateral or asymmetric narrowing of the maxilla. Although it might seem that the use of SARPE is limited, it is essential to compare the longterm stability, morbidity of a 2-stage vs a 1-stage procedure, and the psychological impact of 2 procedures on the patient rather than 1 procedure. Proponents of SARPE have also hypothesized that postSARPE orthopedic forces can be applied to the maxilla, since the 2 halves of the maxilla have been loosened. These forces might be valuable in correct in sagittal or vertical discrepancies without additional surgery. This, however, has not been used routinely because the prognosis is uncertain [74].

\section{Periodontal Status}

Muller and Eger and Muller et al recently introduced the concept of periodontal biotype. They pointed out that it is essential to record the thickness of the gingival tissues during clinical evaluation of the periodontium. This is especially important because a thin and delicate gingiva might be prone to recession after traumatic, surgical, or inflammatory injuries. Histologic studies of the supporting tissues around extracted teeth that were initially used as appliance anchors have shown that a strong inflammatory responses during maxillary expansion. Orthodontic tooth movement can have a detrimental influence on the mucogingival complex, especially when the keratinized tissue and underlying bone appear to be thin. Therefore, evaluations of the gingival tissues and the biotype are essential to determine the ability of the tissues to withstand the pressure of OME; otherwise, surgical release of the sutures is needed to remove interferences to maxillary expansion. The selection of the appliance type (number of anchor teeth included or tooth-borne vs bone-borne appliances) might also depend directly on the periodontal biotype. These appliances are discussed in detail below [75].

\section{Orthodontic Considerations and Preparation}

Before sending a patient for a SARPE, the orthodontist must ensure that there is enough space between the roots of the central incisors for a midline split. A periapical or occlusal radiograph should be taken, and the inter radicular bone evaluated. If space is inadequate, preoperative root divergence must be created. To ensure the postoperative and post treatment health of the teeth, the patient should be seen regularly by a periodontist. The gingiva should be healthy between the central incisors. After expansion, a large midline diastema is present, and the central incisors 
should be removed reciprocally at a controlled and slow rate. A similar yet smaller diastema is obtained in patients who undergo OME when the teeth drift to close the space after expansion. No clear protocol is evident from the literature regarding the rate of midline space closure in SARPE patients. Occasionally, clinicians place a pontic tooth in the midline and slowly grind it down on the proximal surfaces to allow for the central incisors to move toward each other [76].

\section{Types of Appliances}

Two types of appliances for RME are most widely recognized in the literature, and the main difference between them is the presence or absence of an acrylic pad close to the palate. The tooth tissue-borne or Haas-type expander possesses this acrylic pad and is assumed to distribute the expanding force between the posterior teeth and the palatal vault. The tooth borne or Hyrax expander does not include the acrylic pad and presumably delivers the force to the maxilla only by means of the appliance-supporting teeth [77]. Even though cephalometric and dental cast investigations have not demonstrated differences between the toothborne and tooth tissue borne expanders, there is no consensus in the literature regarding the differences between the modes of action of the two appliances. The easier hygiene, greater comfort, and prevention of lesions to the palatal mucosa are the advantages of the hygienic appliance. Advantages of the Hyrax expander include its ability to be placed and removed in the orthodontic outpatient clinic without local anesthesia. Dental anchorage gives rise to several complications, including damage to the teeth, possible loss of anchorage, periodontal membrane compression and buccal root resorption, cortical fenestration, and anchorage tooth tipping and segmental tipping [78]. On the other hand, the palatal acrylic expander is stated to be the only appliance that might lead to a significant expansion of the maxillary base [79, 80]. Moreover, the absence of an acrylic pad is stated to allow relapse of the orthopedic effect during the retention stage. Because the tooth-borne expander maintains only the dental arch expansion, the "bone would move through the teeth". No evidence has confirmed such assumptions. Garib et al., found that tooth-borne and tooth tissue borne expanders tended to produce similar orthopedic effects and the tooth tissue-borne expander produced a greater change in the axial inclination of supporting teeth, especially in the first premolars, compared with the tooth-borne expander. To help prevent the dental complications, several bone borne devices (distractors) have been developed. These distractors are placed directly on the palatal bone during surgery. They are claimed to avoid several of the problems associated with the Hyrax expander including damage to the teeth, periodontal membrane compression and buccal root resorption, cortical fenestration, skeletal relapse, and anchorage tooth tipping [81]. The major advantage of the bone-borne devices is that the forces are acting directly to the bone at the mechanically desired level
[82], which prevents dental tipping and keeps segmental tipping to a minimum. The therapeutic spectrum is widened to include periodontally compromised or toothless patients as well as those with vertical growth pattern [83]. Bone-borne devices have several disadvantages, including a risk of damaging the roots of the dentition during placement of the devices, risk of loosening of the module or the abutment plates, and the need to remove the distractor under local anesthesia in the outpatient clinic after the consolidation period [84]. It has been suggested that the relapse is greater when a tooth-borne device is used. An explanation for this might be the tipping of the elements due to the toothborne fixation of the Hyrax expander. Another contributing factor maybe the tipping of the maxillary segments instead of parallel expansion due to the different position of the tooth-borne and bone-borne distractors relative to the "center of resistance", the area where the maxillary halves are still connected to the skull after the corticotomy, the pterygoid region. The results of the study of Koudstaal et al., show that there is no significant difference between the two groups: the bone-borne versus the tooth-borne distraction. This leads to discarding the working hypothesis that in skeletally matured, non-syndromal patients with transverse maxillary hypoplasia, less tipping of the maxillary segments and increased stability in transverse dimensions at tooth and bone levels are achieved with a bone-borne device compared with a tooth-borne expander in SARME [85].

\section{Activation Frequency and Activation Rate}

Surgeons should not expand the maxilla to its desired width intra operatively as a one-stage procedure, as has been suggested [86]. Instead, this should be done in a slow, controlled fashion over days to weeks, depending on the amount of expansion required. Attempting to achieve all the needed expansion intra operatively is not only a dangerous practice, greatly increasing the chances for the development of untoward fractures, but it violates the concept of a SARME as a technique to achieve distraction osteogenesis. Studying the influence of the activation frequency and activation rate in expanded tissues, Ilizarov has found that $0.5 \mathrm{~mm}$ per day results in premature bone consolidation, hindering the desired distraction. Performing activations with $2.0 \mathrm{~mm}$ per day, the author has observed soft tissue damage and bone pseudoarthrosis. The best results for soft tissues (periosteum, vases, nerves, muscles) and bone were obtained at a rate of $1.0 \mathrm{~mm}$ and a frequency off our times a day [87] (i.e., $0.25 \mathrm{~mm}$ per activation).

\section{Local or General Anesthesia}

Several authors have shown that surgically assisted maxillary expansion can be carried out using only sedatives and local anesthesia when a more conservative surgical techniqueis chosen. General anesthesia is preferred for invasive techniques. General anesthesia is imperative for broader surgical techniques 
that may incur excessive bleeding of the nasal mucosa, the maxillary sinus, the sphenopalatine, or the descending palatine arteries. The SARME may become more involved as the patient ages because the resistance to expansion becomes greater as the sutures become more inter digitated. This can lead to a more involved procedure where more of the maxillary articulations need to be released. The procedure in adults usually requires a general anesthetic and hospitalization, as opposed to being an outpatient procedure in younger patients. For simplified techniques using restricted osteotomies only in the areas of greater resistance, surgically assisted maxillary expansion has proven to be possible with local anesthesia and minimum morbidity. This procedure can be carried out as an ambulatory procedure incurring less surgical time and lower costs $[88,89]$.

\section{Differences in Surgical Techniques}

All of the maxillary articulations and suture lines have been found to contribute in different degrees to the resistance to maxillary expansion. This has led to multiple osteotomy and corticotomy designs for separation of the hemi-maxillas in skeletally mature individuals. Results differ based on the placement of the corticotomies and the timing and placement of the orthodontic devices, but all surgical procedures are more stable than orthodontic expansion alone [90]. Early use of SARME was based on the hypothesis that the palatal suture was the main resistance to expansion and a midpalatal osteotomy was suggested. Isaacson and Ingram $^{44}$ believed that the remaining maxillary articulations were more important sources of resistance to maxillary expansion. Timms and Timms and Vero [36] carry out osteotomies only in the medium palatine suture because they believe it to be the main area of resistance to the lateral expansion of the maxilla. Lines and Bell and Epker thought that the principal regions of resistance to expansion were the frontomaxillary, zygomaticotemporal, zygomaticofrontal, and the zygomaticomaxillary sutures [52]. Kennedy et al., [39] popularized the use of an osteotomy of the zygomaticomaxillary buttress as the major factor in overcoming resistance to maxillary expansion. Osteotomy only in the bilateral lateral wall, from the piriform opening to the maxillary tuberosity, without releasing the pterygoid lamina and with no osteotomy in the medium palatine suture was successfully used by Glassman et al., [49] and Antilla et al., Other authors prefer a combination of both techniques (i.e., osteotomies in the median palatine suture and in the bilateral lateral wall without releasing the pterygoid lamina) because they believe it facilitates the expansion of the maxilla and reduces the possibility of further complications. Some authors prefer subtotal Le Fort I osteotomy associated with median palatine suture osteotomy because they consider the results to be more stable in the long term. According to the study of Shetty et al., the lateral maxillary cuts appeared to decrease the resistance of the maxilla to transverse expansion, as evidenced by increased stress at the various other sutures, particularly the pterygomaxillary articulation, the fronto nasal suture, and along the lateral nasal wall. Separation of the pterygomaxillary articulation was believed to result in a more substantial reduction in the resistance to maxillary expansion because it led to a marked increase in the stresses at distant locations, including the zygomatico frontal suture, the inferior part of the lateral nasal wall, the zygomaticomaxillary suture and posteriorly along the zygomatic arch, and at the supra orbital and frontal bone regions. The fact that there was no appreciable increase in the stresses evidenced in the orbit and in the orbital surface of the sphenoid bone was an important finding after the pterygomaxillary dysjunction. It was the conclusion of Shetty et al., that exclusive use of bilateral zygomaticomaxillary buttress osteotomies to facilitate SARME was inadequate. They believed that analysis of the stress patterns in the analog showed that the midpalatal and pterygomaxillary articulations were the primary anatomic sites of resistance to expansion forces. They therefore thought that complete midpalatal and pterygomaxillary osteotomies were essential to result in predictable maxillary expansion in adults. The greater concentration of stresses noted in the posterior aspect of the midpalatal suture of the analog indicated that this region provides significant resistance to expansion forces. A palatal osteotomy that extends to the posterior aspect of the hard palate was therefore thought to be more appropriate than a more limited anterior osteotomy. Moreover, the study of Chamberl and and Proffit [92], with separation of the pterygoid junction, did not confirm previous reports of a hingetype expansion with SARME, with more expansion anteriorly than posteriorly.

This suggests that changes in recent years in the surgical procedure for SARME, which now includes surgical release of the pterygoid junction, may allow a similar anterior and posterior expansion. The data of the study of these same authors do not support the conclusion of earlier studies of SARME that this procedure produces more stable expansion than do segmental osteotomies. Some authors have confirmed that it is unnecessary to carry out osteotomy in the pterygoid processes $[93,94]$. To minimize the surgical trauma, less invasive procedures combined with osteotomies in the bilateral zygomatic maxillary crest and median palatine suture were performed by Kaban [95] and Pogrel et al., The surgical technique that releases all maxillary joints is preferred by some authors who affirm that the separation of the pterygoid processes, in all cases, is the purpose so as not to reduce the expansion in the posterior region because, contrarily to the maxilla, the sphenoid is a single bone with two articulated processes for each maxilla.

According to Lanigan and Mintz [96], surgeons must give serious consideration to routinely including separation of the pterygomaxillary 
Sharath Kumar Shetty et al., Sch J Dent Sci, Jan, 2021; 8(1): 25-40

articulation as part of a SARME procedure to minimize chances for the development of aberrant fractures. Besides, a greater degree of ossification in the midpalatal synostosis has been noted posteriorly than anteriorly [97]. The pterygomaxillary dysjunction should be carried out via a technique that results in consistent, predictable, safe results, such as with the use of a micro oscillating saw. The use of a curved pterygoid osteotome should be abandoned because its use can result in high pterygoid plate fractures, which have the potential to disrupt the contents of the pterygopalatine fossa and the use of the pterygoid osteotome has been associated with fractures that extend to the skull base and orbit. In the study of Isaacson and Ingram [44], it was noted that a smaller load per activation was produced in the younger patients than in the older teenagers. Because the force values recorded from the expansion device gives an indication of the resistance of the facial skeleton to expansion, this suggests that the facial skeleton increases its resistance to expansion significantly with increasing age and skeletal maturity. Because patients in whom a SARME procedure is carried out tend to be older, a potential exists for even greater forces to be generated, which could be transmitted to distant anatomic sites if the sites of resistance to maxillary expansion are not appropriately released surgically. These observations could stress the need for pterygomaxillary osteotomies. Although simple procedures, such as midpalatal and or zygomaticomaxillary buttress osteotomies, may be sufficient to facilitate maxillary expansion in the vast majority of patients, this may not always be the case, particularly in patients who present with abnormal anatomy. Unfortunately, it is impossible to predict preoperatively which patients will fail to respond successfully to a minimally invasive procedure [98].

\section{SARME and Le Fort I}

The similar stabilities of transverse expansion of the dental arches with SARME and segmental Le Fort I osteotomies provide some insight into the choice between procedures ${ }^{99}$. When only a transverse change is needed, SARME would be the treatment of choice. When a second phase of maxillary surgery to reposition the maxilla vertically or antero posteriorly is required, the routine performance of a preliminary SARME procedure to obtain better transverse stability does not appear to be warranted. Multisegmental osteotomies have been shown to result in greater transverse maxillary instability and relapse after the removal of orthodontic appliances, particularly when used to correct large transverse maxillary discrepancies. Performing a preliminary SARME procedure decreases the risk of aseptic necrosis and relapse, particularly in those patients who have a major transverse discrepancy. An exceptionally narrow maxilla that requires major expansion across the posterior teeth may be an exception. Even though SARME offers many advantages, segmentalized LeFort osteotomies may be indicated in certain circumstances. When transverse discrepancies coexist with vertical or sagittal discrepancies, a segmentalized Le Fort osteotomy should be considered. Patients with moderate transverse discrepancies $(<7 \mathrm{~mm})$, apertognathia, and a severe curve of Spee should be treated with segmental orthodontics and alignment of the segments with a partitioned LeFort osteotomy. Surgically assisted rapid maxillary expansion occurs primarily at the canines and less at the molars, where as the segmental LeFort osteotomy produces more expansion in the molar region than at the canines. The former occurs because the posterior articulations of the maxilla are not separated with SARME. The lateral nasal walls and palatine processes are left intact, limiting posterior expansion [100, 101]. Chamberland and Proffit [92] state that the surgical release of the pterygoid junction may allow a similar anterior and posterior expansion and that relapse in the amount of archwidth increase produced by SARME is comparable to the relapse with other expansion procedures.

\section{Postexpansion Evaluation and Treatment}

A periapical or occlusal radiograph is taken, and the symmetry of the bone should be evaluated. The circumference of the central incisors is gently probed, and the pocket depths compared to presurgical values. Four outcomes are possible:

1. Symmetric bone on the mesial surfaces of both central incisors and the gingival attachment is intact, i.e. the interdental bone was fractured through the palatal suture and equal bone exists on the mesial surfaces of both central incisors; activate the appliance one turn every day and evaluate weekly.

2. Symmetric bone on the mesial surfaces of both central incisors but the gingival attachment is poor. Do not activate appliance until the gingiva is healthy. Activate appliance one turn every other day and evaluate weekly.

3. Asymmetric bony separation with healthy gingival attachment. Activate appliance one turn every other day and evaluate every 48-72 h.

4. Asymmetric bony separation with poor gingival attachment. Do not activate appliance until the gingiva is healthy. Activate appliance one turn every other day and evaluate every 48-72 hour [102]. 


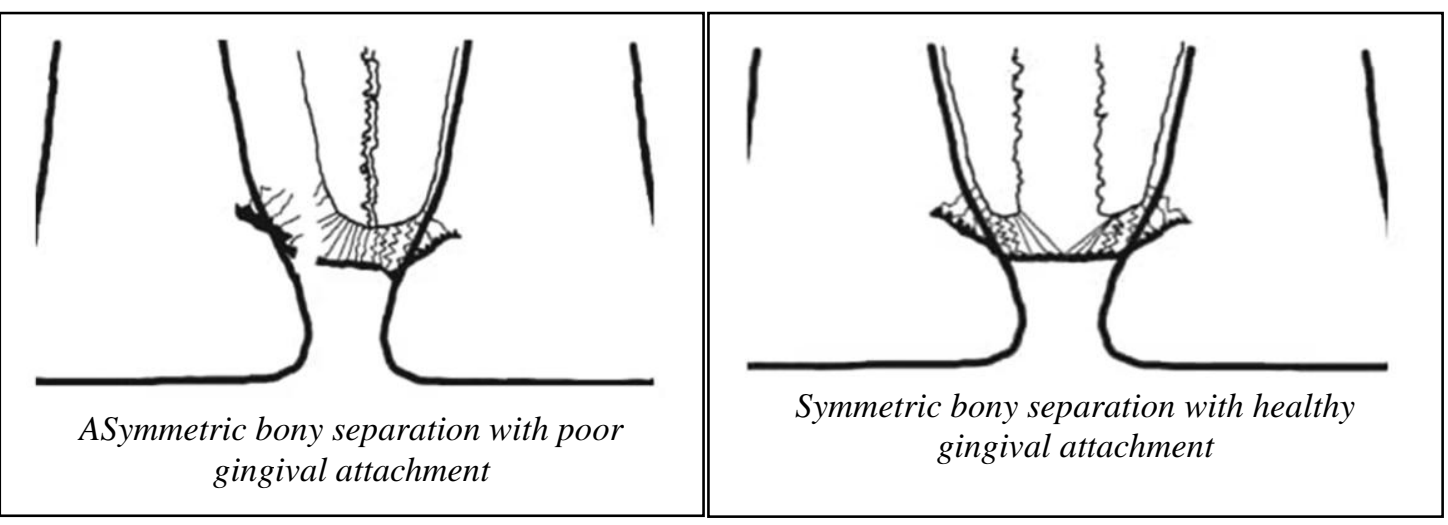

Other Uses of Surgically Assisted Rapid Palatal Expansion

- A morphologically narrow palate has been associated with mouth breathing and altered neuromuscular patterns. The consequences of ventilatory dysfunction are complex and thought to be related to sleeping disorders, including sleep apnea and nocturnal enuresis

- It can be hypothesized that similar associations between MTD in adults and some effects of ventilator dysfunction exist in which SARPE might be useful.

- The recover y of transverse growth discrepancy by surgical and mechanical enlargement produces substantial enlargement of the maxillary apical base and the palatal vault [102].

\section{Treatment Effects on Hard Tissue}

There is some debate over the relative degree of skeletal and dental effects and whether or not expansion occurs evenly throughout the maxilla. Differentiation of dental and skeletal components in the treatment outcome is crucial with respect to stability and relapse [6]. Study models provide limited information about the skeletal effects. Using posteroanterior radiographs, Berger et al., Byloff and Mossaz [38] reported respectively 52 per cent and 24 per cent skeletal effects for SARME and orthodontic treatment. In a radiographic implant study in growing individuals, Krebs reported different effects in various zones of the maxilla after non -surgical orthopaedic expansion. Dental expansion was greater than skeletal expansion and more pronounced anteriorly than posteriorly. Furthermore, there was more expansion in the alveolar process than in the maxillary base. However, Krebs' results should be extrapolated with caution with respect to SARME, because of differences in study populations, age and the additional surgical procedures. Many investigators have tried to verify Krebs results, but their findings have been contradictory. Although a number of reports of the treatment effects of SARME have been published, surprisingly little detailed information exists with reference to long-term stability. In a review of the literature, Koudstaal et al., [33] found no consensus with respect to long-term stability and relapse. Furthermore, apart from the diversity of the surgical and orthodontic procedures, which complicates comparison of treatment outcomes, the sample sizes in previous studies were often too small and/ or the follow up periods were too short. Swennen et al., concluded that there is a lack of appropriate data on long-term follow-up and relapse. In a review of the literature, Lagravere et al., found six long-term studies with follow-up of more than one year [85].

\section{Treatment Effects on Soft Tissue}

Consistent clinical findings after maxillary osteotomies and SARME are changes in soft tissue and a widening of the nose. Previous studies on SARME and its effects on soft tissue have been limited by the methods available at the time for quantifying soft tissue changes hence the reported findings are doubtful. Ngan et al., and Filho et al., used traditional two-dimensional (2-D) lateral cephalograms. Berger et al., used serial frontal photographs and Ramieri et al., utilized laser scanning and 3D morphometry. The major disadvantage of the methods applied in all the above-cited studies is the potential for errors associated with uncertain superimposition $[45,80]$.

\section{Treatment Effects on Nasal Respiration}

During the early 1900's, numerous papers, mostly based on subjective findings, referred to maxillary expansion and its favourable implications for nasal respiration [103]. Brown described the first case in which nasal blockage was "cured" by rapid maxillary expansion. This favourable effect of RME on nasal respiration was later associated with Krebs' radiological findings of an outward displacement of the lateral walls of the nasal cavity. Furthermore, Babacan et al., observed lowering of the palatal vault, lengthening of the nasal septum and lateralization of the inferior nasal turbinates and thereby an improved respiratory pattern. Hershey et al., stated that RME was an effective method of widening the nasal passages and reducing nasal resistance (NAR) from levels associated with mouthbreathing to levels compatible with normal respiration [104]. Timms argued that the anatomical changes at the nostrils correlated with the patients' subjective perception of improved nasal respiration. Niinemma et al., and Subtelny hypothesized that there is a defined breakpoint of nasal resistance which will lead to either 
nose breathing or to mouth-breathing. Vig however, questioned a direct association between nasal obstruction and mouth-breathing and assumed that mouth breathing might be a learned phenomenon that is not attributable solely to nasal obstruction and a narrow maxilla. Timms evaluated the occurrence of respiratory symptoms, albeit in a limited retrospective study of patients with MTD, and found an increase in respiratory disease and an improvement in nasal blockage after palatal expansion. It has been suggested that long-faced individuals with MTD are candidates for respiratory disturbances. A number of studies report that RME can affect the size of the nasal passages and airway resistance, favouring improved nasal respiration [105]. There is a lack of consensus with respect to RME in terms of mode of action, long-term effects and justification as a modality for the treatment of nasal blockage. However, the results of improved respiration after RME should be extrapolated with caution with respect to SARME. Although many RME studies have implied improvement in nasal respiration, the reports did not take into account confounding factors such as growth, age and the effects of the surgical intervention. Few studies have investigated variables related to nasal obstruction in non- growing individuals with MTD or the effects of SARME on nasal patency. Wriedt et al., showed a tendency toward increased nasal volume after SARME and their findings were supported by Babacan et al., However the findings were not significant. Furthermore, the sample sizes were small and there is some uncertainty regarding the methodology [106].

\section{Retention, Stability and Relapse}

The issue of long-term stability and relapse with SARPE has not been studied in detail in the literature. In general, most reports state that surgical expansion is more stable than OME. Some authors recommended that retention is not necessary for SARPE, and the orthodontist can begin orthodontic treatment without a holding phase. Other authors recommended a period of retention after expansion varying from 2 to 12 months [107], The relapse rates for SARPE vary from $5 \%$ to about $25 \% .7$, These rates are significantly lower than the relapse rate of OME, which can be as high as $63 \%$. The high rate of relapse associated with OME is due to its use in skeletally advanced patients [108]. OME is neither predictable nor stable in older patients. In a study by Berger et al., both OME and SARPE were compared in an age-appropriate sample. The OME sample comprised subjects aged 6 to 12 years, and the SARPE group's ages ranged from 13 to 35 years. These authors found no difference in the stability of SARPE and OME. They, however, did not quantify the relapse amount in either group. Most studies on SARPE discussed relapse as an issue that the clinician should be aware of but reported that the incidence of relapse is low. Few studies cite the need to over expand with SARPE. This is especially true for bone-borne appliances; the relapse was subjectively reported to be extremely low [109].

\section{Complications}

Postsurgical complications may arise if the maxilla is insufficiently released or if the expansion device is improperly constructed. Inadequate bony release will be evident if the patient complains of excessive pain and pressure during activation of the appliance. This is due to force transmitted to the fused sutures. If expansion continues without adequate bony release, teeth and alveolar segments will tip and gingival recession will occur on the buccal surfaces [101]. Root resorption with tooth-borne expanders in nongrowing subjects may occur. Vanarsdall stated that even children with maxillary transverse deficiencies are subject to dehiscence of bone and gingival recession following nonsurgical RME [13].

Complications related to the expansion appliance include breakage or loosening of the device, stripping or locking of the screw, and impingement on the palatal mucosa leading to vascular compromise and tissue necrosis. A palatal tissue irritation from impingement of the expansion appliance on the palatal soft tissues may occur, at times leading to frank aseptic pressure necrosis. In a series reported by Lehman and Haas [48], 3 of 56 patients $(5.4 \%)$ had some degree of palatal mucosal ulceration, while 2 of 56 patients $(1.8 \%)$ developed frank pressure necrosis. In a series reported by Alpern and Yurosko [111], severe tissue irritation under the expansion appliance was noted in several cases, and three adult female patients developed palatal soft tissue aseptic necrosis.

In a study of nonsurgical rapid maxillary expansion cases, Timms and Moss [110] showed histologic evidence of external root resorption and pulpal changes, including the laying down of secondary dentin and pulp stones. It is possible that similar changes could occur after SARME.

Intraoperative complications are uncommon with surgically assisted maxillary expansion. Hemorrhage is another complication that has been reported. The most common sources of hemorrhage after maxillary orthognathic surgery include the terminal branches of the maxillary artery, especially the descending palatine or sphenopalatine arteries, the posterior superior alveolar artery, and the pterygoid venous plexus. The risk of intraoperative bleeding, although rare, exists if the pterygoid plates are separated. The descending palatine artery is particularly vulnerable to damage when a SARME is performed with either pterygomaxillary separation or a lateral nasal wall osteotomy [101]. Lanigan [112] states that separation of the pterygoid plates may infrequently cause excessive hemorrhage, thrombosis (which can lead to stroke), and arterio venous fistulae between the carotid sinus and carotid artery. The cause of abnormal bleeding during maxillary orthognathic procedures can be either mechanical disruption of an artery or vein or secondary to a bleeding diasthesis. Turvey and Fonseca 
showed that the mean distance from the most inferior part of the pterygomaxillary junction to the most inferior part of the internal maxillary artery is $25 \mathrm{~mm}$.

With a correctly positioned osteotome during the pterygomaxillary separation, the margin of safety for avoiding direct damage to the maxillary artery from the osteotome should thus be approximately $10 \mathrm{~mm}$ in the adult patient. Damage to the descending palatine artery can also be minimized by limiting the extent of the osteotomy posterior to the piriform rim to $35 \mathrm{~mm}$ in men and $30 \mathrm{~mm}$ in women. It should be noted that these are mean values and do not take into account the range of normal anatomic variation [113].

A dramatic case of life-threatening recurrent haemorrhage was reported by Mehra et al., A case of an orbital compartment syndrome from a retrobulbar hemorrhage, resulting in permanent blindness, has been reported in a 34-year-old woman with transverse maxillary deficiency. A case reported by Lanigan and Mintz [112] of retrobulbar hemorrhage after the use of an expansion device, it is apparent that the forces associated with an expansion device can be transmitted widely within the craniofacial complex, which was demonstrated in details by Shetty et al., These forces have the potential to lead to aberrant fractures that can run to the base of the skull, orbit, and pterygopalatine fossa and could result in injuries to important neurovascular structures [114].

Bilateral lingual nerve injury after a routine and uncomplicated SARME procedure was reported. The authors hypothesize that there was an aberration in the anatomic pathway of the lingual nerve in this patient and that two probable mechanisms could have been responsible for this transient injury: direct injury to the lingual nerve with the pterygomaxillary osteotomies or nerve compression from a hematoma in the pterygomaxillary region.

Shetty et al., [114] have analyzed internal stress responses after SARME using a photoelastic analog of a human skull made from a birefringent cortical bone simulant. The orthopedic forces produced by the Hyrax appliance had deep anatomic effects, with internal stresses being manifested at regions distant from the site of force application. These forces were transmitted into the midface and craniofacial complex along the classic midfacial support struts the nasomaxillary, the zygomaticomaxillary, and the pterygomaxillary buttresses.

With propagation of the split along the midpalatal suture, an immediate alteration in the stress distribution throughout the craniofacial complex was noted. Completion of the midpalatal osteotomy produced a demonstrable increase in the stresses at the zygomaticomaxillary, zygomaticofrontal and frontonasal sutures as well as along the anterior portion of the lateral nasal wall. In addition, there was a concomitant increase in the stresses observed in the orbital region, especially involving the orbital surface of the greater wing of the sphenoid bone.

Still in the experiment conducted by Shetty et al., [114] using the photoelastic analog, failure to separate the pterygomaxillary junction resulted in forces radiating across the pterygoid plates to deeper anatomic structures, including the body and greater wing of the sphenoid bone. A close anatomic relationship exists between the greater and lesser wings of the sphenoid bone, the sphenoid sinuses, and the inferior and superior orbital fissures. The sphenoid sinus is related laterally to the optic nerve as it traverses the optic foramen, the cavernous sinus, and the internal carotid artery. Therefore, sphenoid sinus fractures have the potential to lead to tears in adjacent soft tissue structures, resulting in carotid-cavernous sinus fistulae, injuries to the carotid artery, damage to the optic nerve, or injuries to cranial nerve III, IV, or VI leading to ophthalmoplegia. Palatal tori also complicate SARME. Patients should have the torus removed 4 to 6 months before surgery. However, if removal at the time of SARME is planned, an appliance must be constructed on a model with the torus removed. Care should be taken to assure that the appliance is not impinging on the palatal mucosa and vascular pedicle after torus removal. There should be minimal periosteal stripping when a combined SARME and torus removal are done because a midpalatal incision will be required to remove the torus and this is an area of limited blood supply [101].

\section{CONCLUSIONS}

SARPE is a widely used procedure for the correction of MTD in skeletally mature patients. However, there is sparse information on many issues pertaining to SARPE. There are still no conclusive ways to identify the optimal equilibrium between extensive surgeries for adequate mobilization vs a conservative procedure with minimal complications. Advances in imaging techniques have added another dimension to the evaluation of bone density and surgical manipulation. These can assist in achieving greater precision and help standardize surgical techniques and orthodontic treatment protocols.

\section{REFERENCES}

1. Vanarsdall RL, White Jr RP. Three-dimensional analysis for skeletal problems. The International journal of adult orthodontics and orthognathic surgery. 1994;9(3):159.

2. Betts NJ, Vanarsdall RL, Barber HD, HigginsBarber K, Fonseca RJ. Diagnosis and treatment of transverse maxillary deficiency. The International journal of adult orthodontics and orthognathic surgery. 1995;10(2):75.

3. Menon S, Manerikar R, Sinha R. Surgical management of transverse maxillary deficiency in 
adults. Journal of maxillofacial and oral surgery. 2010 Sep 1;9(3):241-6.

4. Pereira MD, Prado GP, Abramoff MM, Aloise AC, Ferreira LM. Classification of midpalatal suture opening after surgically assisted rapid maxillary expansion using computed tomography. Oral Surgery, Oral Medicine, Oral Pathology, Oral Radiology, and Endodontology. 2010 Jul $1 ; 110(1): 41-5$.

5. Harvold EP, Chierici G, Vargervik K. Experiments on the development of dental malocclusions. American journal of orthodontics. 1972 Jan 1;61(1):38-44.

6. Odont EL. Sucking, chewing, and feeding habits and the development of crossbite: a longitudinal study of girls from birth to 3 years of age. The Angle Orthodontist. 2001 Apr;71(2):116-9.

7. Øgaard B, Larsson E, Lindsten R. The effect of sucking habits, cohort, sex, intercanine arch widths, and breast or bottle feeding on posterior crossbite in Norwegian and Swedish 3-year-old children. American Journal of Orthodontics and Dentofacial Orthopedics. 1994 Aug 1;106(2):1616.

8. Allen D, Rebellato J, Sheats R, Ceron AM. Skeletal and dental contributions to posterior crossbites. The Angle Orthodontist. 2003 Sep 1;73(5):515-24.

9. de Silva Fo OG, Boas CV, Capelozza LF. Rapid maxillary expansion in the primary and mixed dentitions: a cephalometric evaluation. American Journal of Orthodontics and Dentofacial Orthopedics. 1991 Aug 1;100(2):171-9.

10. Haas AJ. The treatment of maxillary deficiency by opening the midpalatal suture. The Angle Orthodontist. 1965 Jul 1;35(3):200-17.

11. Bishara SE, Staley RN. Maxillary expansion: clinical implications. American journal of orthodontics and dentofacial orthopedics. 1987 Jan 1;91(1):3-14.

12. Jacobs JD, Bell WH, Williams CE, Kennedy III JW. Control of the transverse dimension with surgery and orthodontics. American journal of orthodontics. 1980 Mar 1;77(3):284-306.

13. Vanarsdall RL, White RP, Jr. Three-dimensional analysis for skeletal problems, Int $\mathrm{J}$ Adult Orthodon Orthognath Surg, 1994; 9(3):159.

14. Latham RA, Burston WR. The postnatal pattern of growth at sutures of the human skull. Dent Pract. 1966: 17:61-67.

15. Krogman WM. Studies in growth changes in the skull and face of anthropoids: Ectocranial and endocranial suture closure in anthropoids and old world apes. Am J Phys Anthropol. 1930: 46:315353.

16. Sicher H. Oral anatomy. ed. 4, St. Louis, 1965, the C.V. Mosby Company: 71-75.

17. Persson M. Structure and growth of facial sutures. Odontol Revy. 1973: 24(6).
18. Scott JH. Dentofacial development and growth. oxford, 1967, Pergamon Press: 89-93

19. Melsen B. Palatal growth studied on human autopsy material.Am J Orthod. 1975: 68:42-54.

20. Öztürk M, Doruk C, Özeç I, Polat S, Babacan H, Biçakci A. Pulpal bloodflow: effects of corticotomy and midline osteotomy in surgically assisted rapid palatal expansion. J CranioMaxillofacial Surg. 2003: 31:97-100.

21. Profitt WR. Contemporary orthodontics, third edition, 2000. Penny Rudolph, Mosby Inc, St Louis, USA.

22. Lefoulon PJ. Orthopédie dentaire. Gazette de l'hôpital. 1839: 111-119.

23. Talma AF. Mémoires sur quelques points fondamentaux de la medicine dentaire. Bruxelles: Tircher. 1852.

24. Angell EH. Treatment of irregularity of the permanent or adult teeth. Dental Cosmos 1860: 1:540-600.

25. Schröder-Benseler. Die kiefererweiterung. Erg ges Zahnhk. 1913: 629.

26. Derichsweiler H. Gaumennatherweiterung, munich: Karl Hanser: 1956.

27. Swennen G, Schliephake H, Dempf R, Schierle H, Malevez C. Craniofacial distraction osteogenesis: a review of the literature. Part 1: clinical studies. International journal of oral and maxillofacial surgery. 2001 Apr 1;30(2):89-103.

28. Yen SL. Distraction osteogenesis: application to dentofacial orthopedics, Semin Orthod, 1997; 3(4):275-83.

29. Codivilla A. On the means of lengthening in lower limbs, the muscles and tissues which are shortened though deformity, American Journal of Orthopaedic Surgery, 1905; 2:353.

30. Cope JB, Samchukov ML, Cherkashin AM. Mandibular distraction osteogenesis: a historic perspective and future directions, Am J Orthod Dentofacial Orthop. 1999; 115(4):448-60.

31. Al-Daghreer S, Flores-Mir C, El-Bialy T. Longterm stability after craniofacial distraction osteogenesis, J Oral Maxillofac Surg, 2008; 66(9):1812-9.

32. Kojimoto HA, Yasui NA, Goto TA, Matsuda S, Shimomura YU. Bone lengthening in rabbits by callus distraction. The role of periosteum and endosteum. The Journal of bone and joint surgery. British volume. 1988 Aug;70(4):543-9.

33. Koudstaal MJ, Van der Wal KG, Wolvius EB, Schulten AJ. The Rotterdam Palatal Distractor: introduction of the new bone-borne device and report of the pilot study. International journal of oral and maxillofacial surgery. 2006 Jan $1 ; 35(1): 31-5$

34. McCarthy JG, Stelnicki EJ, Mehrara BJ, Longaker MT. Distraction osteogenesis of the craniofacial skeleton. Plastic and reconstructive surgery. 2001 Jun 1;107(7):1812-27. 
Sharath Kumar Shetty et al., Sch J Dent Sci, Jan, 2021; 8(1): 25-40

35. Bierenbroodspot F, Wering PC, Kuijpers-Jagtman AM, Stoelinga PJ. Surgically assisted rapid maxillary expansion: a retrospective study. Nederlands tijdschrift voor tandheelkunde. 2002 Aug;109(8):299-302.

36. Timms DJ, Vero D. The relationship of rapid maxillary expansion to surgery with special reference to midpalatal synostosis, Br J Oral Surg. 1981; 19(3):180-96.

37. Bays RA, Greco JM. Surgically assisted rapid palatal expansion: an outpatient technique with long-term stability. J Oral Maxillofac Surg. 1992; 50(2):110-3

38. Byloff FK, Mossaz CF. Skeletal and dental changes following surgically assisted rapid palatal expansion, Eur J Orthod. 2004; 26(4):403-9

39. Kennedy JW, 3rd. Osteotomy as an adjunct to rapid maxillary expansion, Am J Orthod. 1976; 70(2):123-37.

40. Stromberg C, Holm J. Surgically assisted, rapid maxillary expansion in adults. A retrospective long-term follow-up study, J Craniomaxillofac Surg. 1995; 23(4):222-7.

41. Steinhauser EW. Midline splitting of the maxilla for correction of malocclusion. J Oral Surgery. 1972: 30:413-422.

42. Brown GVI. The surgery of oral and facial diseases and malformation. 1938. $4^{\text {th }}$ Ed., London; Kimpton: 507.

43. Heiss J. Ueber die chirugische Untersteutzung der Dehnung im komprimierten Oberkiefer. Deutsch Zahnaeszteblatt. 1954: 8:560.

44. Isaacson RJ, Ingram AH. Forces produced by rapid maxillary expansion I: Forces present during treatment. Angle Orthod. 1964: 34:256-260.

45. Wertz RA. Skeletal and dental changes accompanying rapid midpalatal suture opening. Am J Orthod. 1970: 58:41-66.

46. Steinhauser EW. Midline splitting of the maxilla for correction of malocclusion. J Oral Surgery. 1972: 30:413-422.

47. Steinhauser EW. Midline splitting of the maxilla for correction of malocclusion. J Oral Surgery. 1972: 30:413-422.

48. Lehman JA, Haas AJ. Surgical-orthodontic correction of transverse maxillary deficiency. Clin Plast Surg. 1989: 16:749-755.

49. Glassman AS, Nahigian SJ, Medway JM, Aronowitz HI. Conservative surgical orthodontic adult rapid palatal expansion: sixteen cases. Am J Orthod. 1984: 86:207-213.

50. Mommaerts MY. Transpalatal distraction as a method of maxillary expansion. Brit J Oral Maxillofac Surg. 1999: 37:268-272.

51. Gerlach KL, Zahl C. Transversal palatal expansion using a palatal distractor. J Orofac Orthop. 2003: 64: 443-449.

52. Bell WH, Epker BN. Surgical orthodontic expansion of the maxilla. 1976: 70:517-528.
53. Ilizarov GA. Clinical applications of the tensionstress effect for limb lengthening. Clin Orthop. 1990: 250:8-26.

54. Lines PA. Adult rapid maxillary expansion with corticotomy, Am J Orthod, 1975; 67(1):44-56.

55. Bell WH, Epker BN. Surgical orthodontic expansion of the maxilla. 1976: 70:517-528

56. Woods M, Wiesenfeld D, Probert T. Surgicallyassisted maxillary expansion. Aust Dent J. 1997; 42:38-42.

57. Koudstaal MJ, Poort LJ, van der Wal KG, Wolvius EB, Prahl-Andersen B, Schulten AJ. Surgically assisted rapid maxillary expansion (SARME): a review of the literature. Int $\mathbf{J}$ Oral Maxillofac Surg. 2005; 34:709-14.

58. Dawson PE. New definition for relating occlusion to varying conditions of the temporomandibular joint. J Prosthet Dent. 1995; 74:619-27.

59. Guichet NF. Biologic laws governing functions of muscles that move the mandible. Part I. Occlusal programming. J Prosthet Dent. 1977; 37:648-56.

60. Jacobs JD, Bell WH, Williams CE, Kennedy JW 3rd. Control of the transverse dimension with surgery and orthodontics. Am J Orthod. 1980; 77:284-306.

61. Rakosi T, Jonas I, Graber TM. Color atlas of dental medicine: orthodontic diagnosis. New York: Thieme Medical Publishers; 1993.

62. Redmond WR. Digital models: a new diagnostic tool. J Clin Orthod. 2001; 35:386-7.

63. Lehman JA Jr, Haas AJ, Haas DG. Surgical orthodontic correction of transverse maxillary deficiency: a simplified approach. Plast Reconst Surg. 1984; 73:62-8.

64. Bell WH, Epker BN. Surgical-orthodontic expansion of the maxilla. Am J Orthod 1976; 70:517-28.

65. Ricketts RM. Perspectives in the clinical application of cephalometrics. The first fifty years. Angle Orthod. 1981; 51:115-50.

66. Macchi A, Carrafiello G, Cacciafesta V, Norcini A. Threedimensional digital modeling and setup. Am J Orthod Dentofacial Orthop. 2006; 129:60510.

67. Epker BN, Wolford LM. Transverse maxillary deficiency dentofacial deformities: integrated orthodontic and surgical correction. St Louis: Mosby; 1980.

68. Mossaz CF, Byloff FK, Richter M. Unilateral and bilateral corticotomies for correction of maxillary transverse discrepancies. Eur J Orthod. 1992; 14:110-6.

69. Inoue N, Oyama K, Ishiguro K, Azuma M, Ozaki T. Radiographic observation of rapid expansion of human maxilla. Bull Tokyo Med Dent Univ. 1970; 17:249-61.

70. Melsen B. Palatal growth studied on human autopsy material. A histologic microradiographic study. Am J Orthod. 1975; 68:42-54. 
Sharath Kumar Shetty et al., Sch J Dent Sci, Jan, 2021; 8(1): 25-40

71. Persson M, Thilander B. Palatal suture closure in man from 15 to 35 years of age. Am J Orthod. 1977; 72:42-52.

72. Alden TD, Lin KY, Jane JA. Mechanisms of premature closure of cranial sutures. Childs Nerv Syst. 1999; 15:670-5.

73. Cohen MM Jr. Sutural biology and the correlates of craniosynostosis. Am J Med Genet. 1993; 47: 581-616.

74. Muller HP, Eger T. Masticatory mucosa and periodontal phenotype: a review. Int $\mathbf{J}$ Periodontics Restorative Dent. 2002; 22:172-83.

75. Muller HP, Schaller N, Eger T. Ultrasonic determination of thickness of masticatory mucosa: a methodologic study. Oral Sur Oral Med Oral Pathol Oral Radiol Endod 1999; 88:248-53.

76. Cureton SL, Cuenin M. Surgically assisted rapid palatal expansion: orthodontic preparation for clinical success. Am J Orthod Dentofacial Orthop. 1999; 116:46-59.

77. Haas AJ. Interviews. J Clin Orthod. 1973; 7:227245.

78. Koudstaal MJ, Smeets JB, Kleinrensink GJ, Schulten AJ, van der Wal KG. Relapse and stability of surgically assisted rapid maxillary expansion: an anatomic biomechanical study. J Oral Maxillofac Surg. 2009; 67:10-14.

79. Haas AJ. Rapid expansion of the maxillary dental arch and nasal cavity by opening the midpalatal suture. Angle Orthod. 1961; 31:73-90

80. Haas AJ. Long-term post-treatment evaluation of rapid palatal expansion. Angle Orthod. 1980; 50:189-217.

81. Mommaerts MY. Transpalatal distraction as a method of maxillary expansion. $\mathrm{Br} \quad \mathrm{J}$ Oral Maxillofac Surg. 1999; 37:268-272.

82. Pinto PX, Mommaerts MY, Wreakes G, Jacobs WV. Immediate postexpansion changes following the use of the transpalatal distractor. J Oral Maxillofac Surg. 2001; 59:994-1001.

83. Hansen L, Tausche E, Hietschold V, Hotan T, Lagravère M, Harzer W. Skeletally-anchored rapid maxillary expansion using the Dresden distractor. J Orofac Orthop. 2007; 68:148-158.

84. Koudstaal MJ, Smeets JB, Kleinrensink GJ, Schulten AJ, van der Wal K. Relapse and stability of surgically assisted rapid maxillary expansion: an anatomic biomechanical study. J Oral Maxillofac Surg. 2009; 67:10-14.

85. Koudstaal MJ, Wolvius EB, Schulten AJ, HopWC, van derWal KG. Stability, tipping and relapse of bone-borne versus tooth-borne surgically assisted rapid maxillary expansion; a prospective randomized patient trial. Int $\mathrm{J}$ Oral Maxillofac Surg. 2009; 38:308-315.

86. Messer EJ, Bollinger TE, Keller JJ. Surgicalmechanical maxillary expansion. Quint Int. 1979; 8:13-16.

87. Pogrel MA, Kaban LB, Vargervik K, Baumrind S. Surgically assisted rapid maxillary expansion in adults. Int J Adult Orthod Orthognath Surg. 1992; 7:37-41.

88. Glassman AS, Nahigian SJ, Medway JM, Aronowitz HI. Conservative surgical orthodontic adult rapid palatal expansion: sixteen cases. Am J Orthod. 1984; 86:207213.

89. Messer EJ, Bollinger TE, Keller JJ. Surgicalmechanical maxillary expansion. Quint Int. 1979; 8:13-16.

90. Pogrel MA, Kaban LB, Vargervik K, Baumrind S. Surgically assisted rapid maxillary expansion in adults. Int J Adult Orthod Orthognath Surg. 1992; 7:37-41.

91. Byloff FK, Mossaz CF. Skeletal and dental changes following surgically assisted rapid palatal expansion. Eur J Orthod. 2004; 26:403-409.

92. Chamberland S, Proffit WR. Closer look at the stability of surgically assisted rapid palatal expansion. J Oral Maxillofac Surg. 2008; 66:1895-1900.

93. Northway WM, Meade JB Jr. Surgically assisted rapid maxillary expansion: a comparison of technique response and stability. Angle Orthod. 1997; 67:309-320.

94. Pogrel MA, Kaban LB, Vargervik K, Baumrind S. Surgically assisted rapid maxillary expansion in adults. Int J Adult Orthod Orthognath Surg. 1992; 7:37-41.

95. Kaban LB. Surgical orthodontic correction of transverse maxillary deficiency: a simplified approach-discussion. Plast Reconst Surg. 1984; 73:67-68.

96. Lanigan DT, Mintz SM. Complications of surgically assisted rapid palatal expansion: review of the literature and report of a case. J Oral Maxillofac Surg. 2002; 60:104-110.

97. Persson M, Thilander B. Palatal suture closure in man from 15 to 35 years of age. Am J Orthod. 1977; 72:42-52.

98. Lanigan DT, Guest P. Alternative approaches to the pterygomaxillary dysjunction. Int $\mathbf{J}$ Oral Maxillofac Surg. 1993; 22:131-138.

99. Chamberland S, Proffit WR. Closer look at the stability of surgically assisted rapid palatal expansion. J Oral Maxillofac Surg. 2008; 66:1895-1900.

100. Philips C, Medland WH, Fields HW Jr, Proffit WR, White RP Jr. Stability of surgical maxillary expansion. Int $\mathbf{J}$ AdultOrthod Orthognath Surg. 1992; 7:139-146.

101. Silverstein K, Quinn PD. Surgically-assisted rapid palatal expansion for management of transverse maxillary deficiency. J Oral Maxillofac Surg. 1997; 55:725-727.

102. Cureton SL, Cuenin M. Surgically assisted rapid palatal expansion: Orthodontic preparation for clinical success. Am J Orthod Dentofacial Orthop. 1999; 116:46- 59. 
Sharath Kumar Shetty et al., Sch J Dent Sci, Jan, 2021; 8(1): 25-40

103. Dean LW. The influence of the nose on wideningthe palatal arche, Journal of the American Medical Association, 1909; 52:941-43.

104. Hershey HG, Stewart BL, Warren DW. Changes in nasal airway resistance associated with rapid maxillary expansion, Am J Orthod. 1976; 69(3):274-84.

105. De Felippe NL, Da Silveira AC, Viana G, Kusnoto B, Smith B, Evans CA. Relationship between rapid maxillary expansion and nasal cavity size and airway resistance: short-and longterm effects. American Journal of Orthodontics and Dentofacial Orthopedics. 2008 Sep 1;134(3):370-82.

106. Berretin-Felix G, Yamashita RP, Nary Filho H, Gonales ES, Trindade Jr AS, Trindade IE. Shortand long-term effect of surgically assisted maxillary expansion on nasal airway size. Journal of Craniofacial Surgery. 2006 Nov 1;17(6):10459.

107. Neyt NM, Mommaerts MY, Abeloos JV, De Clercq CA, Neyt LF. Problems, obstacles and complications with transpalatal distraction in noncongenital deformities. J Craniomaxillofac Surg. 2002; 30:139-43.
108. Mew J. Long-term effect of rapid maxillary expansion. Eur J Orthod. 1993; 15:543.

109. Zahl C, Gerlach KL. Palatal distractor. An innovative approach for palatal expansion. Mund Kiefer Gesichtschir. 2002; 6:446-9.

110. Timms DJ. Effect of rapid maxillary expansion on hearing loss. Angle Orthod. 1997; 67:244-246.

111. Alpern MC, Yurosko JJ. Rapid palatal expansion in adults: with and without surgery. The Angle Orthodontist. 1987 Jul;57(3):245-63.

112. Lanigan DT, Mintz SM. Complications of surgically assisted rapid palatal expansion: review of the literature and report of a case. Journal of oral and maxillofacial surgery. 2002 Jan 1;60(1):104-10.

113. Turvey TA, TA T, RJ F. The anatomy of the internal maxillary artery in the pterygopalatine fossa: its relationship to maxillary surgery. J Oral Surg. 1980; 38:92-95.

114. Shetty V, Caridad J, Caputo AA, Chaconas SJ. Biomechanical rationale for surgical-orthodontic expansion of the adult maxilla. Journal of oral and maxillofacial surgery. 1994 Jul 1;52(7):742-9. 